Purpose To assess the budget impact of introducing FCM in the current practise for treating postoperative anaemia in orthopaedic surgery.

Materials and Methods A budget impact model (BIM) was built from a hospital perspective. Study population consisted of patients who underwent total hip or knee replacement in 2011. Costs are estimated by micro-costing for treatment costs and questionnaire for nursing costs. A reference case is based on the present patient case-mix. Simulations consider different substitutions: simulation A $100 \%$ ISC for FCM, simulation B 100\% ISC and 50\% oral iron for FCM and simulation C 100\% ISC and 100\% oral iron for FCM. Oneway sensitivity analysis is applied to simulations.

Results Population: 314 patients (210 women) underwent 327 operations (205 total hip replacements), mean age was 71.6 years. Costs per treatment: oral iron $€ 0.57$, ISC $€ 60.48$, FCM $€ 82.46$ and transfusion $€ 431.13$ (no patient received erythropoietin treatment during hospitalisation). Average costs per patient: reference case $€ 161.63$, simulation $A € 169.83$, simulation $B € 195.93$ and simulation $C € 219.85$. Total costs per year: reference case $€ 44124.20$, simulation A $€ 46364.85$ (+5\%), simulation B $€ 53488.94$ (+21\%) and simulation C $€ 60018.12$ (+31\%). Discussion: BIM is very sensitive to variations in transfusion rate, moderately sensitive to variations in treatment costs and insensitive to variations in nursing costs. Economically, simulation A is feasible for many patients, simulation $\mathrm{B}$ is feasible, but simulation $\mathrm{C}$ is not.

Conclusions FCM will be added to the hospital formulary. A further study is needed to define substitution modalities in the real-life situation. BIM has contributed to this decision-making process.

No conflict of interest.

\section{OHP-079 TUMOR NECROSIS FACTOR BLOCKERS IN RHEUMATOLOGY; CONVENTIONAL VERSUS OFF-LABEL DRUG DOSAGE}

doi:10.1136/ejhpharm-2013-000276.452

'MA Aranguren Redondo, 'G Lopez Arzoz, 'MB Irastorza Larburu, 'G Liceaga Cundin, ${ }^{2} \mathrm{O}$ Maiz Alonso, 'I Aguirre Zubia, 'J Belzunegui Otaño, 'MP Bachiller Cacho, 'MI Fernandez Gonzalez, 'O Valbuena Pascual. 'Donostia University Hospital, Pharmacy, San Sebastián, Spain; ${ }^{2}$ Donostia University Hospital, Rheumatology, San Sebastián, Spain

Background Drug dosage modifications are a common clinical practise regarding Tumor Necrosis Factor (TNF) blockers, using posologies not specified on the authorised product information summary. This practise has a significant financial impact on the healthcare system.

Purpose To revise and investigate actual drug dosages in our Hospital's rheumatology service for conventional TNF blockers.

Materials and Methods The Pharmacy Service analysed the internal data record for rheumatology patients treated during April 2012 and for at least one year with infliximab (IFX), etanercept (ETN) or adalimumab (ADA). Off-label indications were excluded. Therapeutic indication, initial and current posology were recorded.

Results Number of patients by drug;

\begin{tabular}{rrr}
\hline IFX & ETN & ADA \\
\hline 128 & 152 & 121 \\
\hline
\end{tabular}

Number of patients by indication:

\begin{tabular}{llll}
\hline RA & AS & PA & JIA \\
\hline 208 & 109 & 79 & 5 \\
\hline
\end{tabular}

RA: Rheumatoid arthritis, AS Ankylosing spondylitis, PA psoriatic arthritis, JIA: Juvenile idiopathic arthritis

Regarding posology, 261 patients (65\%) were on a conventional dose (CD), $93(23 \%)$ on a reduced dose (DR) and $47(12 \%)$ on an increased dose (DI)
Percentage of patients by drug on CD, DR or DI was;

\begin{tabular}{llll}
\hline Treatment/posology & CD & DR & DI \\
\hline IFX & $33 \%$ & $35 \%$ & $32 \%$ \\
ETN & $79 \%$ & $21 \%$ & - \\
ADA & $82 \%$ & $14 \%$ & $4 \%$ \\
\hline
\end{tabular}

Percentage of patients by indication was;

\begin{tabular}{llll}
\hline Indication/posology & CD & DR & DI \\
\hline RA & $65 \%$ & $19 \%$ & $16 \%$ \\
AS & $59 \%$ & $32 \%$ & $9 \%$ \\
PA & $72 \%$ & $24 \%$ & $4 \%$ \\
JIA & $80 \%$ & $20 \%$ & - \\
\hline
\end{tabular}

Conclusions Only $65 \%$ of patients using TNF blockers on rheumatology use a CD while a quarter of them have a reduced posology.

Infliximab is the drug that requires more dosage modifications, on almost $2 / 3$ of patients.

$\mathrm{AS}$ and PA are the indications that allow more DR.

Drug dosage revisions at the end of the first year of treatment allow an important number of patients to reduce their dose while controlling their disease and it is a relevant efficacy instrument.

No conflict of interest.

\section{OHP-080 USE OF CHEMOTHERAPY NEAR THE END OF LIFE}

doi:10.1136/ejhpharm-2013-000276.453

A Martín, S Ibañez, C Blázquez, BM Muñoz, MT Franco, Al Fernández, M Rodriguez, R Pérez, C Encinas. Hospital General, Pharmacy, Ciudad Real, Spain

Background Appropriately timed cessation of chemotherapy is integral to the patient's quality of life.

Purpose To describe and evaluate the use of chemotherapy in cancer patients in their last days of life.

Materials and Methods Retrospective observational study that included all cancer patients who died in our hospital in 2011. Information sources used were: a) Mambrino for the age, date of death of the patient and clinical charts; b) Oncofar to record the type of cancer, the last cycle of intravenous (IV) chemotherapy received, the historic administration, lines of treatment and the percentage of the last dose received; c) APD-Athos to review data from the patient's hospital stay and outpatient oral cytostatics dispensing. We collected for each patient their demographics, pharmacotherapy, the temporal interval between the last chemotherapy administration and death of the patient and the number of days in hospital one month before death.

Results A total of 94 patients (30\% female) died in 2011 in our hospital. Of these, 10 patients didn't receive chemotherapy, 10 received IV chemotherapy combined with oral, 4 received oral chemotherapy alone and 70 IV chemotherapy alone. Tumours with the highest number of deaths were non-small cell lung cancer (21), head and neck cancer (11) and colorectal cancer (10). The most common last chemotherapy regimens were combinations of carboplatin (16) (especially with pemetrexed and paclitaxel), gemcitabine (11) (mostly alone), combinations of cisplatin (9), paclitaxel (9) (alone or combined with carboplatin) and monoclonal antibodies (9) (in $67 \%$ combined with bevacizumab); the most frequent oral chemotherapy drugs were erlotinib (4) and temozolomide (3). Of the 80 patients who received IV chemotherapy, $27.5 \%$ (22) received chemotherapy in the last 14 days of life, another $27.5 \%$ (22) received chemotherapy between 15 and 30 days before death, $21.25 \%$ (17) between 31 and 60 days, 13.75\% (11) between 61 and 90 days and $10 \%$ (8) more than 90 days before death. In addition, 14\% (12) 